RESEARCH ARTICLE

\title{
Airway-directed gene transfer of interleukin-10 using recombinant Sendai virus effectively prevents post- transplant fibrous airway obliteration in mice
}

\author{
F Shoji ${ }^{1,2}$, Y Yonemitsu ${ }^{1}$, S Okano ${ }^{1}$, I Yoshino ${ }^{2}$, K Nakagawa ${ }^{1}$, Y Nakashima ${ }^{1}$, M Hasegawa ${ }^{3}$, \\ $\mathrm{K}$ Sugimachi ${ }^{2}$ and K Sueishi ${ }^{1}$ \\ ${ }^{1}$ Division of Pathophysiological and Experimental Pathology, Department of Pathology, Graduate School of Medical Sciences, Kyushu \\ University, Fukuoka, Japan; ${ }^{2}$ Department of Surgery and Science, Graduate School of Medical Sciences, Kyushu University, Fukuoka, \\ Japan; and ${ }^{3}$ DNAVEC Research Inc., Tsukuba, Ibaraki, Japan
}

Bronchiolitis obliterans (BO) after lung transplantation prevents a satisfactory prognosis, and recent studies suggested that interleukin-10 (IL-10) gene transfer to distant organs could inhibit $B O$ in rodent models. Although delivery of the therapeutic gene to a local airway would be favored to minimize systemic effects, current limitations include lower gene transfer efficiency to airway epithelium. As recombinant Sendai virus (SeV) can produce dramatically efficient gene transfer to airway epithelium, we determined if SeV-mediated IL-10 gene transfer to the local airway would inhibit bronchial fibrous obliteration in murine tracheal allografts. Administration of cyclosporine $A(C s A)$ significantly promoted not only recovery of the injured airway epithelium but also SeVmediated IL-10 expression (CsA-versus CsA+=228 \pm 78 versus $3627 \pm 1372 \mathrm{pg} / \mathrm{graft}$ with $\left.5 \times 10^{7} \mathrm{pfu}\right)$, thereby suggesting the requirement of epithelia for efficient gene transfer. Even at the highest expression, no significant leakage of IL-10 was evident in the systemic circulation, and the induction of interferon- $\gamma$ was completely diminished on day 7 by IL-10 gene transfer. As a result, luminal loss was significantly prevented in allografts treated with SeV-IL-10 (luminal opening, all control groups: 0\% respectively, and SeV-IL-10 $5 \times 10^{7}$ pfu: $25.7 \pm 10.5 \%$ ), an effect that was enhanced by short-term CsA treatment (SeV-IL-10 $5 \times 10^{7}$ pfu with CsA: $63.7 \pm 12.7 \%$ ). We propose that SeV is a useful vector that can target airway epithelium to prevent BO avoiding putative systemic effect.

Gene Therapy (2003) 10, 213-218. doi:10.1038/sj.gt.3301847

Keywords: bronchiolitis obliterans (BO); recombinant Sendai virus (SeV); SeV-IL-10; local gene transfer

\section{Introduction}

Lung transplantation can be effective for patients with end-stage pulmonary diseases; however, post-transplantation bronchiolitis obliterans (BO), a form of chronic graft rejection, occurs in approximately $50 \%$ of recipients surviving over 3 months after receiving a graft. Although improvement in surgical techniques, careful immunosuppression and postoperative management have improved the outcome after lung transplantation, ${ }^{1,2}$ preventive treatment for $\mathrm{BO}$ has not been available.

The heterotopic tracheal transplantation model in rodents has been used in laboratory studies. ${ }^{3-5}$ A recent study showed that during development of airway obliteration, a strong Th1 response in allografts was observed whereas no significant changes in Th2 response was reported, and another study showed that an immunosuppressant could inhibit BO in this model, suggesting that the Th1 response against tracheal allografts is likely to be critical in the development of BO. ${ }^{6,7}$ Interleukin-10 (IL-10), an anti-inflammatory

Correspondence: Y Yonemitsu, Division of Pathophysiological and Experimental Pathology, Department of Pathology, Graduate School of Medical Sciences, Kyushu University 3-1-1 Maidashi, Higashi-ku, Fukuoka 812-8582, Japan

Received 26 February 2002; accepted 21 June 2002 cytokine, is a molecule that modulates cellular and hormonal immunity against an allograft by suppressing the expression of IL-2, IL-4, and interferon- $\gamma$ $($ IFN- $\gamma) \cdot)^{8,9}$

Several studies on experimental gene therapy using Il10 to prevent post-transplant fibrous airway obliteration were also reported. A study demonstrated that intramuscular administration of an adenovirus encoding IL10 (Ad-IL-10) prevented post-transplant fibrous airway obliteration in a rat model, as a result of increased circulating IL-10 protein levels. ${ }^{10}$ Although this encouraging study showed the proof of principle of IL-10 gene therapy to prevent post-transplant fibrous airway obliteration, it remained unclear whether increased concentrations of the immunosuppressant IL-10 in systemic circulation are relevant in a clinical setting. In our pilot study, mice with intramuscular IL-10 gene transfer showed increased mortality associated with high circulating IL-10 protein over 10-100 ng/ml (unpublished data). As BO is localized at the lower conducting airway, airway-directed gene transfer can be considered in attempts to avoid systemic effects. However, the low rate of airway gene transfer using currently available vectors, including adenovirus, is hazardous to deliver IL10 gene directly to the airway epithelium.

We recently reported that recombinant Sendai virus $(\mathrm{SeV})$, a new gene transfer agent, produce log-orders- 
high gene transfer efficiency throughout the respiratory tract, as compared to currently available vectors, including adenovirus. ${ }^{11}$ In the present study, we determined if SeV-IL-10 would inhibit luminal occlusion in a murine post-transplant fibrous airway obliteration model by airway-directed gene transfer of IL-10 without increasing blood levels.

\section{Results}

Natural course of epithelial lining in tracheal allografts To clarify if allogenic responses in tracheal explants might differ among strains of mice, we histologically checked six different syngenic (C57BL/6J to C57BL/6J, $n=5$, Balb/c to Balb/c, $n=5$, and $\mathrm{C} 3 \mathrm{H}$ to $\mathrm{C} 3 \mathrm{H}, n=5)$ or allogenic (C57BL/6J to Balb/c, $n=5$, Balb/c to C57BL/6J, $n=5, \mathrm{C} 57 \mathrm{BL} / 6 \mathrm{~J}$ to $\mathrm{C} 3 \mathrm{H}, n=5, \mathrm{C} 3 \mathrm{H}$ to $\mathrm{C} 57 \mathrm{BL} / 6 \mathrm{~J}, n=5$, Balb/c to $\mathrm{C} 3 \mathrm{H}, n=5$, and $\mathrm{C} 3 \mathrm{H}$ to $\mathrm{Balb} / \mathrm{c}, n=5$, respectively) donor-recipient pairs on 3, 7, 28 days after tracheal implantation. All tracheal tissues for syngenic and allogenic implantation showed a complete loss of epithelial lining on day 3 (data not shown), probably due to ischemic damage. On day 7, isografts showed almost complete coverage of the regenerative squamous-type epithelium (Figure 1a-A), while epithelial lining of allografts was extensively lost or was partly replaced by squamous epithelium-like regenerative epithelium (Figure 1a-B). In the later phase on day 28, isografts showed complete recovery of the differentiated respiratory epithelium and were filled with mucinous materials (Figure 1a-C). On the other hand, allografts showed complete loss of the luminal space which was occupied with proliferation of spindle-shaped cells associated with loose connective tissue (Figure 1a-D). These histological findings were consistent in all donor-recipient pairs, suggesting that tissue response to allogenic grafts did not largely depend on the strains of mice; therefore, the following experiments were done using $\mathrm{C} 57 \mathrm{BL} / 6 \mathrm{~J}$ to Balb/c (allogenic) and Balb/c to Balb/c (syngenic). a $\quad 7$ days after grafting

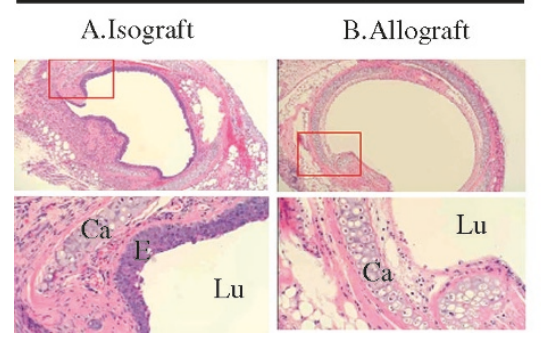

28 days after grafting

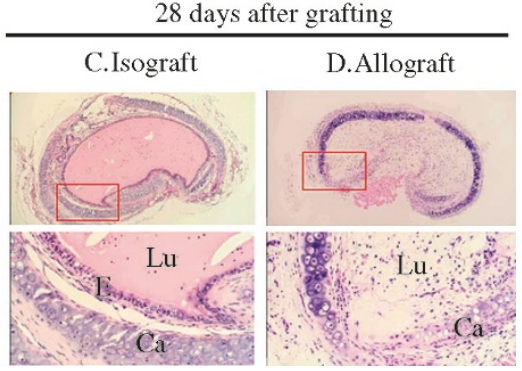

Allografts

b

B.CsA

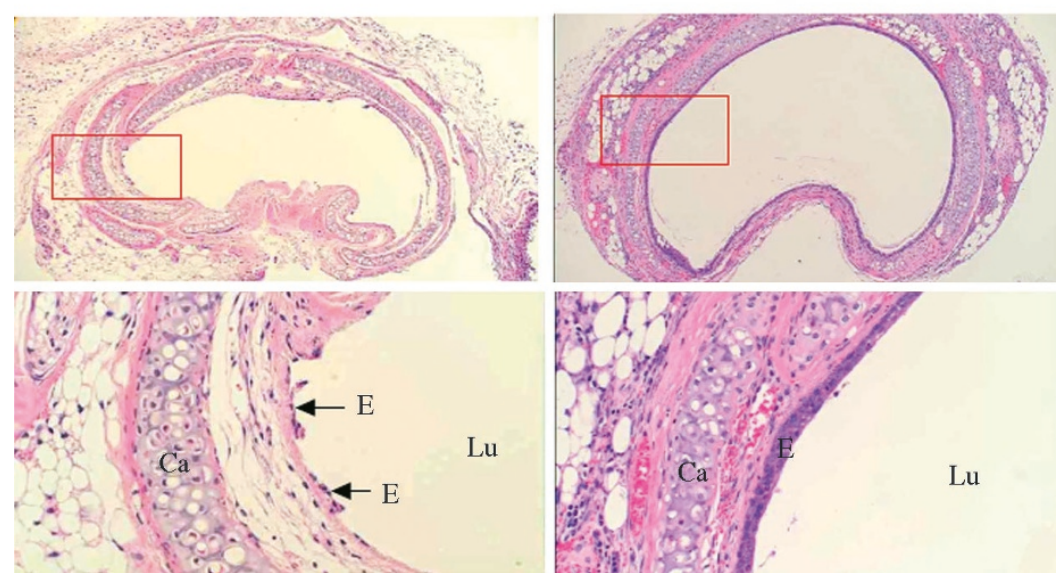

Figure 1 (a) Early $(A$ and $B)$ and late (C and D) change in histopathology of tracheal isograft (donor and recipient: Balb/c) and allograft (donor: C57BL/6; recipient: Balb/c). Lower panels are a high-powered view of the squared area of each upper panel. Lu: lumen; Ca: cartilage, E: epithelial layer. Hematoxylineosin staining. Original magnification: upper panels, $\times 26.5$; lower panels, $\times 106$. A. Tracheal isograft 7 days after transplantation. Note that the luminal surface is lined with squamous-type regenerative epithelium. B. Tracheal allograft 7 days after transplantation. Note that the lining epithelium is lost extensively. C. Tracheal isograft 28 days after transplantation. The luminal space is completely opened and filled with secretion produced by respiratory epithelium. D. Tracheal allograft 28 days after transplantation. The lining epithelium is completely lost and the lumen is occupied with proliferation of spindle-shaped cells associated with loose connective tissue.(b) Effect of short-term intraperitoneal administration of cyclosporine A (CsA: $25 \mathrm{mg} / \mathrm{kg} /$ day for 8 days) on histopathology (A: PBS injected control; B: CsA) and on SeV-mediated IL-10 gene transfer efficiency (C). A and B. Histological findings of lining epithelium in allograft 7 days after transplantation. Lower panels are a high-powered view of the squared area of each upper panel. A PBS-treated allograft shows extensive loss of tracheal epithelium, except for partial coverage of fragmented epithelial cells (arrows). B. Histopathological findings of Cs Atreated allograft. More than $90 \%$ of the luminal surface is covered by the squamous-type regenerative epithelium. Lu: lumen; Ca: cartilage; E: epithelial layer. Hematoxylin-eosin staining. Original magnification: upper panels, $\times 26.5$, lower panels, $\times 106$. 
Short-term treatment of cyclosporine $A$ accelerates epithelial lining as well as airway-directed SeVmediated gene transfer efficiency

Next, we tested the effect of the short-term treatment of cyclosporine A (CsA) on the epithelial lining and SeVmediated gene transfer efficiency. CsA treatment $(25 \mathrm{mg} / \mathrm{kg} /$ day) initiated 1 day prior to tracheal implantation was continued daily for 8 days and then withdrawn on the day before virus administration (day 7).

Untreated allografts showed extensive loss of epithelium (Figure $1 \mathrm{~b}-\mathrm{A}$ ), as seen in a representative result shown in Figure 1a-B, while CsA-treated allografts had an over $90 \%$ epithelial lining (Figure 1b-B). We then assessed the effect of short-term treatment of CsA on SeV-IL-10 gene transfer. As shown in Table 1, IL-10 expression was markedly increased by CsA treatment which suggested that CsA-mediated acceleration of epithelial lining dramatically enhanced SeV-mediated gene transfer. Under condition of CsA treatment, a dosedependent increase of IL-10 expression was also apparent, as shown in Table 1.

\section{Time course of SeV-mediated IL-10 expression and its effect on endogenous IFN- $\gamma$ expression}

As a subsequent experiment, we examined murine IL-10 protein levels in allografts and serum on 2, 7, and 14 days following the luminal administration of SeV-IL-10.

Table 1 Intratracheal IL-10 protein expression of CsA-treated or CsA-untreated allografts 2 days after intratracheal injection of SeVIL-10, and significant dose-dependent increase of gene transfer efficiency

\begin{tabular}{lccc}
\hline $\begin{array}{l}\text { CsA } \\
\text { treatment }\end{array}$ & $\begin{array}{c}\text { No. of } \\
\text { animals }(n)\end{array}$ & $\begin{array}{c}\text { Virus tilter } \\
(p f u / 50 \mu l)\end{array}$ & $\begin{array}{c}\text { Murine IL-10 level } \\
(p g / g r a f t)\end{array}$ \\
\hline No & 3 & 0 & $<5$ \\
Yes & 3 & 0 & $<5$ \\
No & 6 & $5 \times 10^{7}$ & $228 \pm 78.3$ \\
Yes & 6 & $2 \times 10^{6}$ & $12.3 \pm 5.4$ \\
Yes & 6 & $1 \times 10^{7}$ & $401.9 \pm 255.3$ \\
Yes & 6 & $5 \times 10^{7}$ & $3626.7 \pm 1371.9^{\mathrm{a}}$ \\
\hline
\end{tabular}

${ }^{\text {a }} P<0.01$ versus other groups.
As shown in Figure 2a, large amounts of intratracheal IL-10 protein in allografts transfected with SeV-IL-10 $\left(5 \times 10^{7} \mathrm{pfu}\right)$ were evident 2 days after gene transfer $(n=9,3517.7 \pm 951.8 \mathrm{pg} / \mathrm{graft})$ and values decreased 7 days after gene transfer $(n=5,5.3 \pm 2.3 \mathrm{pfu} / \mathrm{graft})$. On the other hand, intratracheal IL-10 protein in allografts treated mice with PBS or SeV-lacZ was not significantly detected. Serum IL-10 protein in all mice was also not detected at all time points (data not shown).

Intratracheal expression of Th1-cytokine, IFN- $\gamma$, was subsequently assessed using the same samples (Figure $2 b$ ). On day 2 after gene transfer, both allografts which injected SeV-IL-10 and SeV-lacZ showed significantly elevated IFN- $\gamma$ level (IL-10: $17.3 \pm 6.5 \mathrm{ng} /$ graft, $n=3$, and lacZ: $51.9 \pm 28.9 \mathrm{ng} /$ graft, $n=3$, respectively), while no significant expression was found in grafts treated with PBS, which suggested that the cause of early elevation of IFN- $\gamma$ might be related to vector-mediated induction of the Th1 response and not to allogenic antigens. The allogenic induction of IFN- $\gamma$ in PBS-treated grafts was significant on day 7, and allografts treated with SeV-lacZ also showed high IFN- $\gamma$ levels. At this time, IL-10 gene transfer completely diminished the expression of endogenous IFN- $\gamma$, thereby indicating successful inhibition of Th1 response due to both allogen and vector infection.

\section{Effects of IL-10 gene transfer on the obstructive pathology of post-transplant fibrous airway obliteration in mice}

Finally, we tested the effects of airway-directed IL-10 gene transfer. The experimental protocol is shown in Figure 3, typical histological findings are given in Figure 4 , and the results are summarized in Figure 5.

All allografts with PBS or transfected with SeV-lacZ showed complete luminal occlusion with associated myofibrous tissue, regardless of short-term CsA treatment. On the other hand, allografts transfected with SeVIL-10 showed a preserved lining of squamous-type epithelium and significant inhibition of luminal occlusion, as compared with findings in other groups $(P<0.05)$. This effect was significantly enhanced by CsA treatment.
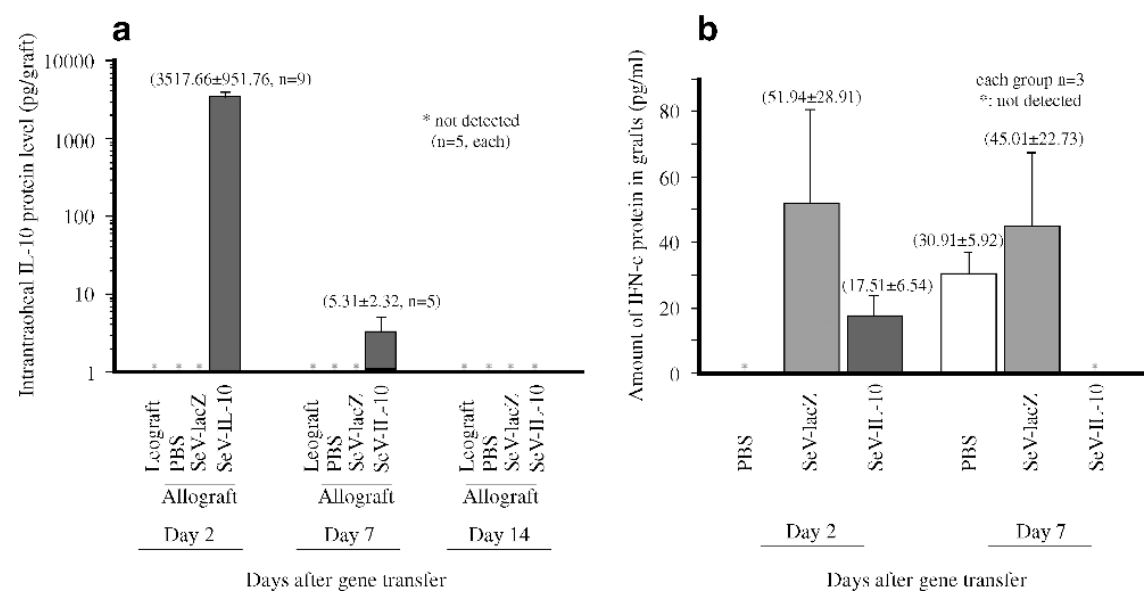

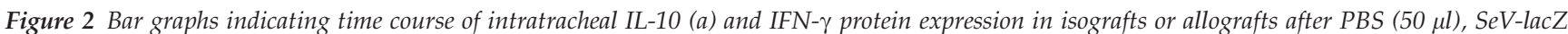
$\left(5 \times 10^{7} \mathrm{pfu} / 50 \mu \mathrm{l}\right)$, or SeV-IL-10 $\left(5 \times 10^{7} \mathrm{pfu} / 50 \mu \mathrm{l}\right)$ injection. At each time point, the grafts were harvested and subjected to enzyme-linked immunosorbent assay. 


\section{a Control Groups}

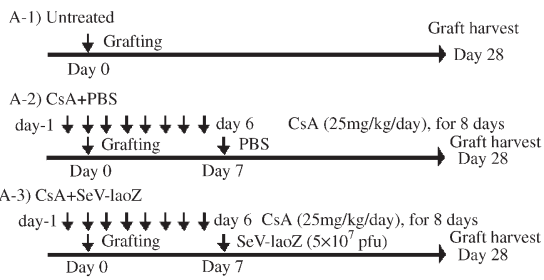

b IL-10 Group

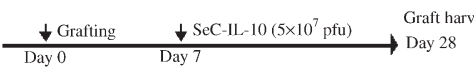

\section{CsA+IL-10 Groups}

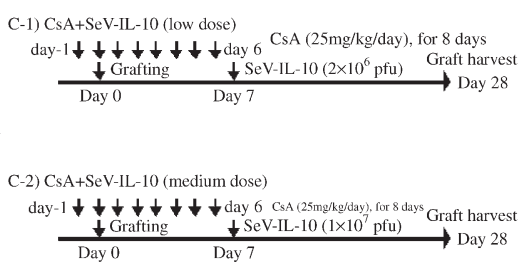

C-3) CsA+SeV-IL-10 (high dose)

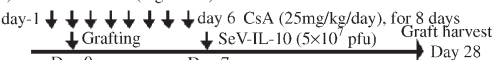

Figure 3 Experimental protocol.

28 days after transplantation

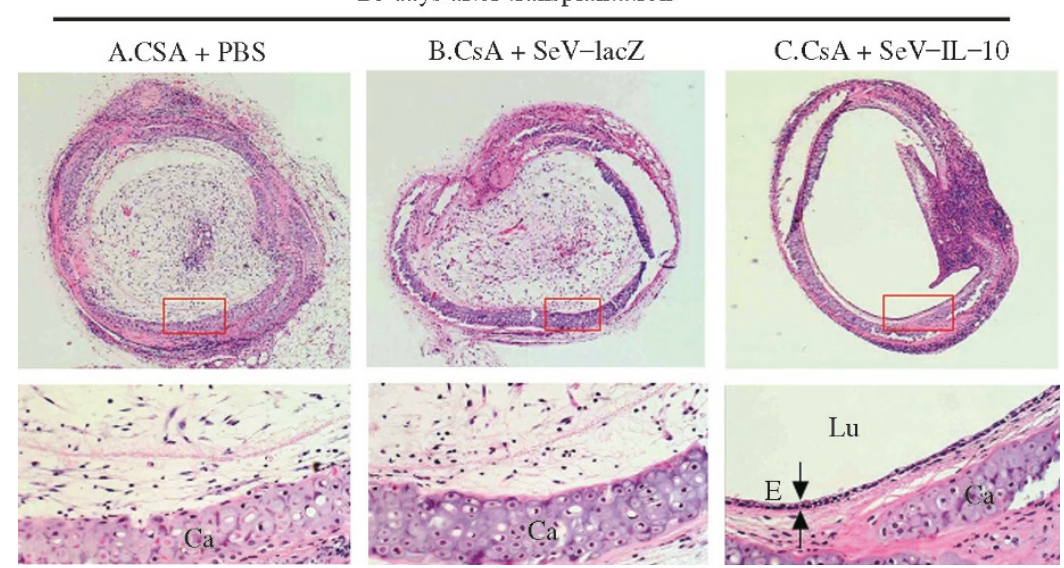

Figure 4 Histological findings of allografts 28 days after transplantation. A and B Histopathological findings of CsA-treated tracheal allografts injected with PBS (A) or SeV-lacZ (B). Both allografts show complete luminal occlusion with myofibrous tissue. C. Histophathological findings of CsA-treated tracheal allografts injected with SeV-IL-10. The luminal loss is well prevented and the luminal surface is lined with squamous-type regenerative epithelium (arrows). Lower panels are a high-powered view of the squared area of each upper panel. Lu: lumen, Ca. cartilage; E: epithelial layer. Hematoxylin-eosin staining, Original magnification: upper panels, $\times 26.5$, lower panels, $\times 106$.

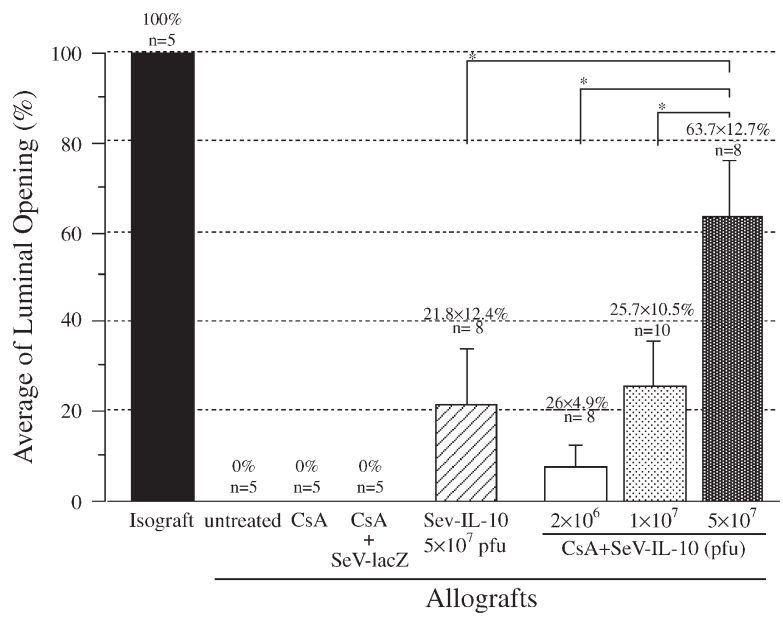

Figure 5 Average of luminal opening in each group. Bar graphs indicating average of the luminal opening in each group. In allografts, the group that was SeV-Il-10 $\left(5 \times 10^{7} \mathrm{pfu}\right)$ injected after CsA treatment showed significant inhibition of luminal occlusion compared with other groups. $\left({ }^{*} P<0.05\right)$.

\section{Discussion}

Few experimental studies on gene therapies for orthotopic lung transplant have been documented. As technical difficulty may be one reason, a heterotopic tracheal transplantation model is used for research on transplant fibrous airway obliteration after lung transplantation. Using this model, we investigated if local SeV-mediated IL-10 gene transfer targeted to the luminal airway epithelium might prevent luminal obstruction in murine heterotopic tracheal allografts. Key findings obtained in this study were: (1) short-term administration of CsA accelerated not only regeneration of the airway epithelium, but also SeV-mediated gene transfer efficiency (2) exogenously transduced IL-10 completely diminished local Th1 cytokine INF- $\gamma$ expression, without any significant leakage to the systemic circulation; and (3) airway-directed IL-10 gene transfer prevented luminal loss of allografts, this effect being significantly enhanced by short-term CsA administration. To our knowledge, this is the first report indicating the significant therapeutic effects of airway-directed IL-10 gene transfer regarding development of post-transplantation fibrous obliteration, and suggests the usefulness of our novel vector; recombinant $\mathrm{SeV}$ can be considered for use in a clinical setting.

Because of the requirement of a simple technique and histological findings similar to the case of human disease, a heterotopic tracheal transplantation model in rodents has been frequently used in laboratory studies 
on BO. Although the relevance of this model has been discussed, immune response-mediated development of the post-transplant fibrous airway obliteration in this model is considered worthwhile of study. Boehler et al reported that Th1 cytokines were upregulated in rat model, ${ }^{6}$ and in a subsequent study they also showed that intramuscular administration of adenovirus encoding IL-10 (Ad-IL-10) inhibited the luminal occlusion of allografts via increased protein levels in the bloodstream. ${ }^{10}$ Although these studies are important to show the 'proof of principle' regarding the role of Th1 cytokines and IL-10 in the development of $\mathrm{BO}$, possible safety concerns in a clinical setting may arise because of increased levels of IL-10 in the systemic circulation. IL-10, an immunosuppressive cytokine, promotes Th2-type cytokines and inversely inhibits Th1-type cytokines and inversely inhibits Th1-type cytokines, including IFN- $\gamma \cdot{ }^{8,9}$ IL-10 also has a significant role down-regulating major histocompatibility complex class II molecules and inhibits antigen-presenting cell functions and production of tumor necrosis factor (TNF)- $\alpha$ and $-\beta$, IL-12, B7 expression and intercellular adhesion molecule-1 (ICAM-1) gene transcription. ${ }^{12-16}$ These broad immunosuppressive effects of IL-10 might be systemically harmful for recipients, with respect to infectious diseases. In this regard, airway-targeted local gene delivery seems more likely to be relevant in preventing $\mathrm{BO}$ in a clinical setting.

We have previously reported the dramatically high gene transfer potential of $\mathrm{SeV}$ vector to airway epithelium in vivo, ${ }^{11}$ and this characteristic was considered to be useful for local IL-10 gene delivery to treat BO. At that time, however, requirement of airway epithelium in allografts was not known concerning efficient gene transfer. Since King et al reported that immunosuppression using CsA significantly reduced development of obliteration in tracheal allografts, ${ }^{3}$ we used CsA in the present study and found that CsA treatment had a beneficial effect not only for the epithelial lining but also on SeV-mediated gene transfer efficiency. These findings show that CsA preserved the lining epithelium that is needed for efficient transfection of SeV-IL-10. Since CsA and other immunosuppressants are clinically available drugs, the current protocol can be applied to clinical use with minor modification.

A question raised in this study is why IFN- $\gamma$ was markedly diminished on day 7 when IL-10 was already down-regulated, as shown in Figure 3b, while suppression of IFN- $\gamma$ was not evident at the highest IL-10 level on day 2. One possible explanation is that 2 days was too early for IL-10 to suppress functions of inflammatory cells, including natural killer cells, monocytes/macrophages, and T-lymphocytes, all of which could produce IFN- $\gamma$ and transmigrate against virus-infected cells. It may be that accumulation of expressed IL-10 suppressed the activity of these cells later, resulting in downregulation of IFN- $\gamma$ on day 7 .

Limitations of the current study are the technical difficulty assessing the long-term effect of locally delivered IL-10. Tracheal allografts collapsed in 2 months due to destruction of tracheal cartilage in this mouse model, even where sufficient CsA was given (unpublished observation). Also, as shown in Figure 3, the current $\mathrm{SeV}$ system produces a foreign gene in only 2 weeks. Future studies using different models and improved vectors persistently express a foreign gene.

In summary, we here demonstrate that airway-directed expression of IL-10 protein could efficiently prevent luminal occlusion of tracheal allografts, and this effect was markedly enhanced by short-term administration of CsA, suggesting a clinically relevant regimen. We propose that SeV-mediated gene transfer can be developed as a safe and effective therapeutic method relevant to clinical use.

\section{Materials and methods}

\section{Gene transfer vectors}

Recombinant SeVs were constructed, as described. ${ }^{17-20}$ In brief, $18 \mathrm{bp}$ of spacer sequence $5^{\prime}-(\mathrm{G})$-CGGCCGCAGATCTTCACG-3' with a Not1 restriction site were inserted between the $5^{\prime}$ nontranslated region and the initiation codon of the nucleoprotein (N) gene. This cloned SeV genome also contained a self-cleaving ribosome site from the antigenomic strand of the hepatitis delta virus. The entire cDNA-coding E. coli lacZ with a nuclear localizing signal (for SeV-nls-lacZ) and murine IL-10 (for SeV-IL-10) were amplified by PCR, using primers with a Not1 site and new sets of SeV E and $S$ signal sequence tags for an exogenous gene, and then inserted into the Not1 site of the cloned genome. The entire length of the template $\mathrm{SeV}$ genomes, including exogenous genes, was arranged in multiples of six nucleotides (so-called 'rule of six'). ${ }^{21}$ Template $\mathrm{SeV}$ genomes with an exogenous gene and plasmids encoding $\mathrm{N}, \mathrm{P}$, and L proteins (plasmid pGEM-N, pGEM-P, and pGEM-L) were complexed with commercially available cationic lipids, and then co-transfected with vaccinia virus vT7-3 into CV-1 or LLMCK cells. Forty hours later, the cells were disrupted by three cycles of freezing and thawing and injected into the chorioallantoic cavity of 10day-old embryonated chicken eggs. Subsequently, the virus was recovered and the vaccinia virus eliminated by a second propagation in eggs. Virus titer was determined by hemagglutination assay, using chicken red blood cells, ${ }^{22}$ and viruses were stored at $-80^{\circ} \mathrm{C}$ until use. The murine IL-10 cDNA for constructing SeV-IL-10 was obtained from American Type Culture Collections (clone 68027, Manassas, VA, USA)

\section{Animals}

A total of 229 pathogen-free male C57BL/6J, Balb/c, and C3H mice (6-8 weeks old, Charles River grade) were purchased from KBT Oriental (Tosu, Saga, Japan). All animal experiments were done using approved protocols and in accordance with recommendations for the proper care and use of laboratory animals by the Committee for Animal and Recombinant DNA Experiments at Kyushu University.

\section{Experiment protocol}

Experimental groups and experimental protocols are summarized in Figure 3. Untreated control $(n=20)$ and some of the treated groups were daily given cyclosporine A (CSA) intraperitoneally at $25 \mathrm{mg} / \mathrm{kg} /$ day for 8 days, as started from day 1 before transplantation. On day 0 , the tracheae from donor mice were transplanted into the subcutaneous tissue of recipient mice, as described. ${ }^{3}$ 
Seven days after transplantation, subcutaneous tissue was incised, grafts were exposed and these lumens were filled with $50 \mu \mathrm{l}$ of PBS or of various titers of vectors (Figure 3). Two 7, 14 and 21 days after gene transfer, mice were killed and grafts and sera were collected.

\section{IL-10 and IFN- $\gamma$ protein analysis}

2,7 , and 14 days after gene transfer, grafts and serum samples from each group were analyzed using commercially available ELISA kits and as instructed by the manufacturer (R\&D Systems, INC., USA).

\section{Histochemistry}

Samples of grafts were fixed in buffered $10 \%$ neutral formalin solution for $24 \mathrm{~h}$. After embedding in paraffin, samples were cut at $5 \mu \mathrm{m}$ thickness and stained with hematoxylin and eosin (H\&E).

\section{Evaluation of luminal occlusion of grafts}

Luminal occlusion was evaluated as described. ${ }^{5}$ In brief, luminal occlusion was defined as the area containing tissue inside of a cartilage ring divided by the total inner area of a cartilage ring. The inner area of cartilage ring includes not only tracheal lumen but the submucosal and epithelial tissue. Luminal occlusion in the isograft measured using this method was 26.7\%; therefore we determined the \% opening by (luminal occlusion in isograft/luminal occlusion in samples) $\times 100$.

\section{Statistical analysis}

All values are expressed as the mean \pm s.e.m. MannWhitney $U$-test was used to analyze data and a value of $P<0.05$ was considered to have statistical significance.

\section{Acknowledgements}

We thank H Fujii and $\mathrm{R}$ Hashimoto for technical assistance.

\section{References}

1 Hosenpud JD et al. The registry of the International Society for Heart and Lung Transplantation: fourteenth official report-1997. J Heart Lung Transplant 1997; 16: 691-712.

2 Sundaresan $S$ et al. Prevalence and outcome of bronchiolitis obliterans syndrome after lung transplantation. Ann Thorac Surg 1995; 60: 1341-1346.

3 King MB et al. Cyclosporine reduces development of obliterative bronchiolitis in a murine heterotopic airway model. Transplantation 1997; 63: 528-532.

4 Hertz MI et al. Reproduction of the obliterative bronchiolitis lesion after heterotopic transplantation of mouse airways. Am J Pathol 1993; 142: 1945-1951.
5 Yamada A et al. Blocking the CD28-B7 T-cell costimulatory pathway abrogates the development of obliterative bronchiolitis in a murine heterotopic airway model. Transplantation 2000; 69: 743-749.

6 Boehler A et al. Upregulation of T-helper 1 cytokines and chemokine expression in post-transplant airway obliteration. Am J Respir Care Med 1999; 159: 1910-1917.

7 Ross DJ et al. Lung allograft dysfunction correlates with gammainterferon gene expression in bronchoalveolar lavage. J Heart Lung Transplant 1999; 18: 627-636.

8 Bromberg JS. IL-10 immunosuppression in transplantation. Curr Opin Immunol 1995; 7: 639-643.

9 Mosmann TR, Sad S. The expanding universe of T-cell subsets: Th1, Th2 and more. Immunol Today 1996; 17: 138-146.

10 Boehler A et al. Adenovirus-mediated interleukin-10 gene transfer inhibits post-transplant fibrous airway obliteration in an animal model of bronchiolitis obliterans. Hum Gene Ther 1998; 9: 541-551.

11 Yonemitsu $\mathrm{Y}$ et al. Efficient transfer to airway epithelium using recombinant Sendai virus. Nat Biotechnol 2000; 18: 970-973.

12 de Waal Malefyt $\mathrm{R}$ et al. IL-10 and v-IL-10 strongly reduce antigen-specific human $\mathrm{T}$ cell proliferation by diminishing the antigen-presenting capacity of monocytes via downregulation of class II MHC expression. J Exp Med 1991; 174: 915-924.

13 D'Andrea A et al. Interleukin 10 (IL-10) inhibits human lymphocyte interferon gamma production by suppressing natural killer cell stimulatory factor/IL-12 synthesis in accessory cells. J Exp Med 1993; 178: 1041-1048.

14 Ding L et al. IL-10 inhibits macrophage costimulatory activity by selectively inhibiting the up-regulation of B7 expression. J Immunol 1993; 151: 1224-1234.

15 Nicod LP et al. Interleukin-10 decreases tumor necrosis factor alpha and beta in alloreaction induced by human lung dendritic cells and macrophages. Am J Respir Cell Mol Biol 1995; 59: 559565.

16 Song $\mathrm{S}$ et al. Interleukin-10 inhibits interferon-gamma induced intercellular adhesion molecule-1 gene transcription in human monocytes. Blood 1997; 89: 4461-4469.

17 Kato A et al. Initiation of Sendai virus multiplication from transfected cDNA or RNA with negative or positive sense. Genes Cells 1996; 1: 569-579.

18 Kato A et al. The paramyxovirus, Sendai virus, V protein encodes a luxury function required for viral pathogenesis. EMBO J 1997; 16: 578-587.

$19 \mathrm{Yu} \mathrm{D}$ et al. Sendai virus-based expression of HIV-1 gp 120: reinforcement by the V(-) version. Genes Cells 1997; 2: 457-466.

20 Sakai $Y$ et al. Accommodation of foreign genes into the Sendai virus genome: sizes of inserted genes and viral replication. FEBS Lett 1999; 456: 221-226.

21 Kolakofsky D et al. Paramyxovirus RNA synthesis and the requirement for hezamer genome length: the rule of six revisited. J Virol 1998; 72: 891-899.

22 Yonemitsu Y, Kaneda Y. Hemagglutinating virus of Japanliposome-mediated gene delivery to vascular cells. In: Molecular Biology of Vascular Diseases. Methods in Molecular Medicine. Baker AH (ed): Humana Press: Clifton, 1999, pp 295-306. 